\title{
Non-Amyloid Approaches to Disease Modification for Alzheimer's Disease: An EU/US CTAD Task Force Report
}

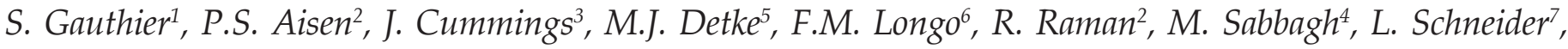 \\ R. Tanzi ${ }^{8}$, P. Tariot ${ }^{9}$, M. Weiner ${ }^{10}$, J. Touchon ${ }^{11}$, B. Vellas ${ }^{12}$ and the EU/US CTAD Task Force*
}

* EU/US/CTAD TASK FORCE: Susan Abushakra (Framingham); John Alam (Boston); Sandrine Andrieu (Toulouse); Anu Bansal (Simsbury); Monika Baudler (Basel); Joanne Bell (Wilmington); Mickaël Beraud (Zaventem); Tobias Bittner (Basel); Samantha Budd Haeberlein (Cambridge); Szofia Bullain (Basel); Marc Cantillon (Gilbert); Maria Carrillo (Chicago); Carmen Castrillo-Viguera (Cambridge); Ivan Cheung (Woodcliff Lake); Julia Coelho (San Francisco); Daniel Di Giusto (Basel); Rachelle Doody (South San Francisco); John Dwyer (Washington); Michael Egan (North Wales); Colin Ewen (Slough); Charles Fisher (San Francisco); Michael Gold (North Chicago); Harald Hampel (Woodcliff Lake); Ping He (Cambridge); Suzanne Hendrix (Salt Lake City); David Henley (Titusville); Michael Irizarry (Woodcliff Lake); Atsushi Iwata (Tokyo); Takeshi Iwatsubo (Tokyo); Michael Keeley (South San Francisco); Geoffrey Kerchner (South San Francisco); Gene Kinney (San Francisco); Hartmuth Kolb (Titusville); Marie Kosco-Vilbois (Lausanne); Lynn Kramer (Westport); Ricky Kurzman (Woodcliff Lake); Lars Lannfelt (Uppsala); John Lawson (Malvern); Jinhe Li (Gilbert); Mark Mintun (Philadelphia); Vaidrius Navikas (Valby); Gerald Novak (Titusville); Gunilla Osswald (Stockholm); Susanne Ostrowitzki (South San Francisco); Anton Porsteinsson (Rochester); Ivana Rubino (Cambridge); Stephen Salloway (Providence); Rachel Schindler (New York); Hiroshi Sekiya (Malvern); Dennis Selkoe (Boston); Eric Siemers (Zionsville); John Sims (Indianapolis); Lisa Sipe (San Marcos); Olivier Sol (Lausanne); Reisa Sperling (Boston); Andrew Stephens (Berlin); Johannes Streffer (Braine-l'Alleud); Joyce Suhy (Newark); Chad Swanson (Woodcliff Lake); Gilles Tamagnan (New Haven); Edmond Teng (South San Francisco); Martin Tolar (Framingham); Martin Traber (Basel); Andrea Vergallo (Woodcliff Lake); Christian Von Hehn (Cambridge); George Vradenburg (Washington); Judy Walker (Singapore); Glen Wunderlich (Ridgefield); Haichen Yang (North Wales); Wagner Zago (San Francisco); Jennifer Ann Zimmer (Indianapolis) Thomas Zoda (Austin)

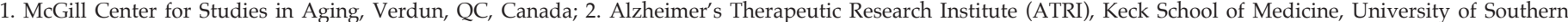

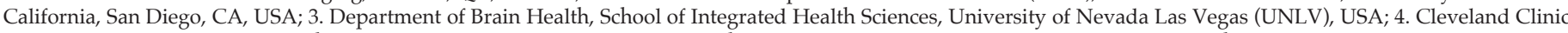

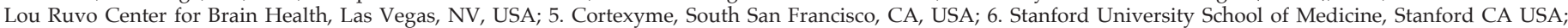

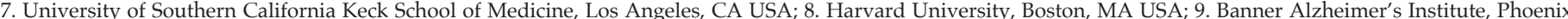

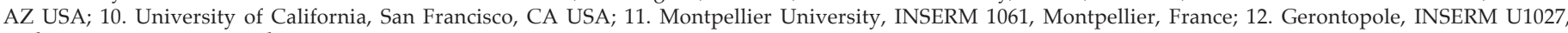
Alzheimer's Disease Research and Clinical Center, Toulouse University Hospital, Toulouse, France

Corresponding Author: Serge Gauthier, McGill Center for Studies in Aging, Verdun QC, Canada, serge.gauthier@mcgill.ca

Published online April 6, 2020, http://dx.doi.org/10.14283/jpad.2020.18

\begin{abstract}
While amyloid-targeting therapies continue to predominate in the Alzheimer's disease (AD) drug development pipeline, there is increasing recognition that to effectively treat the disease it may be necessary to target other mechanisms and pathways as well. In December 2019, The EU/US CTAD Task Force discussed these alternative approaches to disease modification in $\mathrm{AD}$, focusing on tau-targeting therapies, neurotrophin receptor modulation, anti-microbial strategies, and the innate immune response; as well as vascular approaches, aging, and non-pharmacological approaches such as lifestyle intervention strategies, photobiomodulation and neurostimulation. The Task Force proposed a general strategy to accelerate the development of alternative treatment approaches, which would include increased partnerships and collaborations, improved trial designs, and further exploration of combination therapy strategies.
\end{abstract}

Key words: Alzheimer's disease, dementia, tau, tauopathy, neurotrophins, neuroinflammation, lifestyle intervention, photobiomodulation, neurostimulation, geroscience.

\section{Introduction}

$\mathbf{F}$ ollowing a discussion on lessons learned from clinical trials of amyloid-based therapies for Alzheimer's disease (AD) (1), on December 4, 2019, the EU/US CTAD Task Force turned their attention to alternative approaches for disease modification. These strategies do not negate the validity of the amyloid hypothesis; indeed, recently discovered genetic evidence continues to support the centrality of amyloid in the neurodegenerative processes that lead to AD (2-4). However, genetic and other studies point to additional mechanisms and pathways both upstream and downstream of amyloidogenesis, which may provide druggable therapeutic targets with potential for disease modification.

Neuropathological and imaging studies confirm the complexity and heterogeneity of AD (5) Mixed pathologies are evident in most individuals with a clinical diagnosis of AD (6), and in early clinical studies of amyloid-targeting drugs, a significant proportion of trial participants were shown to have no detectable amyloid. Nonetheless, among putative disease-modifying AD drugs in clinical trials, $40 \%$ target amyloid either with small molecules or immunotherapies. Another $18 \%$ target tau. Other mechanisms targeted for disease modification include neuroprotection, anti-inflammatory effects, growth factor promotion, and/or metabolic effects (7). Additional trials are underway assessing non-pharmacological approaches to treat $\mathrm{AD}$, including lifestyle interventions and neurostimulation. 


\section{Anti-tau therapies}

The microtubule-associated protein tau (MAPT, commonly referred to as tau) is the main constituent of the neurofibrillary tangles that are one of the two primary pathological hallmarks of AD. Its normal function is to stabilize microtubules and thus regulate intracellular trafficking, but in $\mathrm{AD}$ and other tauopathies, the protein undergoes post-translational modifications that lead to the development of a variety of oligomeric species, tangles, and neuropil threads that may be deposited as aggregates in specific brain regions, disrupting normal cytoskeletal function and protein degradation pathways (8). In the human brain, six isoforms of tau are present, which are classified as either $3 \mathrm{R}$ or $4 \mathrm{R}$ tau based on the number of repeat domains. Approximately equal levels of $3 \mathrm{R}$ and $4 \mathrm{R}$ tau are expressed in the normal brain; however, 3R:4R tau imbalances are seen in brains of individuals with tauopathies. In AD, isoform imbalances vary across brain regions and disease progression.

Unlike levels of amyloid beta protein $(\mathrm{A} \beta)$, which correlate poorly with cognition, tau levels are associated with both neurodegeneration and cognitive deficits (9). Tau pathology has been shown to follow a characteristic progression pathway in the brain, starting in areas responsible for learning and memory before spreading to cortical areas involved in other cognitive functions (10).

The complex progression of tau pathological events provides multiple potential opportunities for intervention. Anti-tau drugs in development target tau expression, aggregation, degradation, protein modifications (e.g. phosphatase modifiers, kinase inhibitors), microtubule stabilization, and extracellular tau inter-neuronal spread (8). As of February 2019, clinical trials were underway for 17 tau-targeting drugs - seven small molecules and 10 biologics (7). Only one drug, LMTX (TRx0237) - a reduced form of methylene blue, and a tau protein aggregation inhibitor -- is currently being tested in a Phase 3 trial in early AD at $8-16 \mathrm{mg} /$ day doses versus placebo (NCT03446001). This trial follows two Phase 3 trials in mild and mild to moderate AD (NCT01689246, NCT01689233) and a trial in behavioral variant FTD (NCT01626378) with higher doses, which showed negative results in the primary analysis of clinical efficacy. Biogen has a Phase 2 study underway of the anti-tau agent BIIB092 (gosuranemab) in participants with $\mathrm{MCI}$ due to $\mathrm{AD}$ or mild $\mathrm{AD}$ (NCT03352557). Phase 2 studies in biologically defined populations are also being conducted. For example, Roche/Genentech is conducting two Phase 2 studies of the anti-tau monoclonal antibody semorinemab in participants with prodromal or probable AD confirmed by amyloid positron emission tomography (PET) or cerebrospinal fluid (CSF) testing (NCT03828747). Clinical trials of anti-tau therapeutics have been conducted in other tauopathies, although two recent Phase 2 studies of anti-tau monoclonal antibody therapies (Abbvie's
AABV-8E12 and Biogen's gosuranemab) in participants with progressive supranuclear palsy (PSP) were recently terminated for lack of efficacy (NCT2985879 and NCT03068468, respectively). Non-clinical studies of innovative anti-tau therapies are underway, such as a study that uses engineered tau-degrading intrabodies to target intracellular tau (11).

It is also theoretically possible that early anti-amyloid intervention may attenuate or even preclude downstream effects on tau. That is, non-tau-based treatments could have implications for tau and tangles.

Several challenges face developers of tau-based therapeutics. For tau reduction approaches, it is not known how much reduction is needed, how quickly and safely it can be accomplished, when different interventions might be effective during the course of the disease, and how long drug levels must be maintained to get an effect. Tau biology is complicated with numerous fragments and post-translational modifications associated with tauopathies, yet it remains unclear which tau species are toxic. Moreover, the targets, mechanisms and cellular locations through which such tau species promote degeneration remain to be identified. These issues make the design of clinical trials especially complicated and highlight the need for better tau biomarkers. Recent progress made in the development of tau ligands for PET may improve the efficiency of clinical trials, since tau-PET enables early diagnosis and tracking of disease progression, identifying individuals at risk for faster cognitive decline, and rapidly assessing pharmacodynamic effects of treatments (12). Plasma levels of total tau (t-tau) and neurofilament light (NfL) have been developed as biomarkers of neurodegeneration (13). Still needed are biomarkers that distinguish 3R from $4 \mathrm{R}$ tau and that quantify the many different tau species.

\section{Neurotrophic strategies}

The neurodegeneration that occurs in AD results from a complicated molecular and biochemical signaling network, likely triggered by $\mathrm{A} \beta$ and eventually leading to synaptic dysfunction, loss of dendritic spines, and neurite degeneration (14). Growth factors called neurotrophins regulate neuronal survival, development, and function by binding to cell surface receptors. The signaling networks regulated by these receptors have extensive overlap with those associated with neurodegeneration and modulation of neurotrophin receptors has thus been proposed as a potential therapeutic strategy (15). The Longo lab and others have zeroed in on the p75 neurotrophin receptor (p75NTR) as a therapeutic target for AD. Their working hypothesis, supported by human genomic and proteomic data, along with animal studies is that the p75NTR modulates the complex AD degenerative signaling network and that downregulating its signaling renders oligomeric A $\beta$ unable to promote degeneration $(16,17)$.

Longo and colleagues have developed small molecule 
ligands that bind to p75NTR, activate survival-promoting signaling, and prevent $A \beta$-induced neurodegeneration and synaptic impairment (18). One molecule in particular, LM11A-31, has been shown to block $A \beta$-induced tau phosphorylation, misfolding, oligomerization and mislocalization; reverse late-stage spine degeneration; reverse synaptic impairment; prevent microglial dysfunction; and in wildtype mice suppress age-related basal forebrain cholinergic neuron degeneration (18-20). There is evidence that dendritic spine preservation is associated with cognitive resilience (21).

A Phase 2a pilot study sponsored by PharmatrophiX Inc. and funded in part by the National institute on Aging (NIA) and the Alzheimer Drug Discovery Foundation is underway, testing oral LM11A-31 in participants with mild-to-moderate AD and amyloid positivity assessed by CSF A $\beta$ screening (NCT03069014). With an expected completion in the third quarter of 2020, the trial will assess safety and tolerability as well as cognitive, clinical, biomarker, and imaging exploratory endpoints. LM11A-31 may be effective in other disorders such as Huntington's disease (22), diabetes-induced macular oedema (23), and traumatic brain injury (24).

\section{Anti-microbial and anti-inflammatory strategies}

Neuropathological studies of the AD brain show not only amyloid plaques and tau-based tangles but neuroinflammation as well. Indeed, according to the innate immune hypothesis, plaques, tangles, and neuroinflammation orchestrate an innate immune response that has evolved to protect the brain against microbial infection, with A $\beta$ itself acting as an antimicrobial peptide (AMP) in the brain $(25,26)$. This hypothesis suggests that subclinical microbial infections in the brain rapidly 'seed' $A \beta$ to trap microbes, and that this process drives $A \beta$ neurotoxicity and opsonization (i.e, an 'eat me' signal for microglia to remove axons and synapses) (25). Tangles form in response to microbe invasion to block neurotropic microbe spread. $\mathrm{AD}$ risk genes are implicated in the innate immune protection hypothesis, which posits that AD-associated genetic risk variants were evolutionarily conserved to keep $A \beta$ deposition, tangle formation, and gliosis/ neuroinflammation on a 'hair trigger' as a means of protecting a subset of the human species in the advent of a major epidemic of brain infection.

The molecular pathways involved in these processes provide multiple potential therapeutic targets, including the use of anti-viral drugs, antibiotics, blockade of toxic microbial products, and immunization for prevention of subclinical infections; secretase inhibitors and immunotherapies to prevent $A \beta$ seeding; kinase or phosphatase inhibitors to prevent the development of pathological forms of tau, and anti-inflammatories to suppress neuroinflammation. Gut microbiota may also play a role in $\mathrm{AD}$ pathogenesis by disrupting neuroinflammation and metabolic homeostasis, thus representing another potential intervention target (27).

One example of a bacterial hypothesis and associated strategy is based on the discovery of the bacterium Porphyromonas gingivalis (Pg), most commonly associated with periodontitis, in the brains of $\mathrm{AD}$ patients. Toxic virulence factors from the bacterium, proteases called gingipains, have been identified in $\mathrm{AD}$ brains, and gingipain levels correlated with tau and ubiquitin pathology. Oral infection of mice with $\mathrm{Pg}$ resulted in brain colonization, increased $A \beta 1-42$, and loss of hippocampal neurons, effects that were blocked by COR388, a small-molecule irreversible lysine- gingipain inhibitor. COR388 significantly lowered markers of inflammation in plasma as well as AD-associated APOE fragments in CSF in a small Phase $1 b$ study in mildmoderate AD patients (28), and a large Phase 2/3 study is underway with an interim readout expected in Q4 2020 and topline data in Q4 2021 (NCT03823404).

A retrospective cohort study showed that Herpes simplex virus (HSV)-infected subjects had a nearly 3 -fold increased risk of AD but that treatment with anti-viral drugs such as acyclovir brought risk to noninfected levels (29). There is an ongoing phase 2 trial of valacyclovir for patients with mild $\mathrm{AD}$ and positive titers for HSV1 and HSV2 (NCT03282916). Trials in AD using doxycycline and minocycline did not show efficacy (30).

Anti-inflammatory strategies are also being pursued. A Phase 2 study underway in participants with late mild cognitive impairment (MCI) or early AD aims to protect neurons against oxidative stress using two small molecule drugs -- tauroursodexycholic acid (TUDCA) and sodium phenylbutyrate -- repurposed by Amylyx Pharmaceutical as AMX0035 (NCT03533257). Yet another Phase 3 study sponsored by AZTherapies, Inc. aims to reduce neuroinflammation by converting microglia from a proinflammatory to phagocytic state to promote clearance of $A \beta$ by using a combination of two marketed drugs, cromolyn and ibuprofen, known as ALZT-OP1 (NCT02547818) (31).

\section{Lifestyle intervention strategies and other non-pharmacological approaches}

Multiple epidemiological studies in Europe, the United States, and Canada investigating an observed decline in the prevalence of dementia in recent years have suggested that dementia may be preventable by targeting lifestyle risk factors such as diabetes, hypertension, obesity, physical inactivity, smoking, depression, low education, and social isolation (32). Clinical studies are now beginning to support this assertion. The Systolic Blood Pressure Intervention Trial --Memory and Cognition in Decreased Hypertension (SPRINT MIND) study suggested that intensive blood pressure control may reduce the risk of probable dementia and mild 
cognitive impairment (MCI), although the results were not statistically significant, in part because the SPRINT trial was terminated early based on the significant benefits of blood pressure control on cardiovascular outcomes. The study may have been underpowered for cognitive endpoints (33). Further study is warranted given that a 10-year study in France showed that hypertension was associated with poorer cognition in middle-aged individuals (34).

Multi-domain strategies have focused on lifestyle factors. For example, the Finnish Geriatric Intervention Study to Prevent Cognitive Impairment and Disability (FINGER) trial demonstrated improved or stabilized cognitive function in participants that adhered to an intervention combining diet, physical exercise, cognitive training, and vascular risk monitoring (35). The Multidomain Alzheimer Prevention Trial (MAPT) tested an intervention combining cognitive and physical intervention along with omega-3 polyunsaturated fatty acid supplementation in frail, non-demented, community dwelling adults $(36,37)$. While MAPT failed to demonstrate significant slowing of cognitive decline, subgroup analyses suggested that individuals with low plasma levels of docosahexaenoic acid (DHA, an omega-3 fatty acid) have more cognitive decline, which appeared to be normalized with omega-3 supplementation(38). The benefits of omega-3 supplementation appeared to be greater in amyloid-positive individuals and in those with increased cardiovascular risk scores $(39,40)$. Based on the results from FINGER, MAPT, and other multidomain intervention studies, many additional studies are planned, including worldwide FINGERS studies (WW-FINGERS), a network of studies throughout the world that are adapting the multidomain strategies of the FINGER trial to different populations (41).

In addition to physical and cognitive activity, other non-pharmacological strategies are being investigated for their potential to slow cognitive decline and prevent dementia. For example, photobiomodulation (PBM) has been shown to be neuroprotective. In animal models PBM improved memory and normalized markers of AD, oxidative stress and neuroinflammation (42). A pilot study is now underway in participants with probable $\mathrm{AD}$ (NCT03405662).

Non-invasive neurostimulation with techniques such as repetitive transcranial magnetic stimulation (rTMS) has been proposed as a treatment for AD (43). Other technological approaches including assistive technologies, smart technologies, and telemedicine may improve the treatment and care of people with AD.

\section{GeroSciences}

Given that aging is the major risk factor for $A D$, therapeutic strategies aimed at the diseases of aging (e.g., frailty) may slow cognitive decline and the development of dementia (44) Considerable research is underway to investigate the relationship between biological aging and neurodegenerative disease. These efforts have coalesced in the emerging field of geroscience (44), which explores whether the physiological hallmarks of aging such as mitochondrial dysfunction, loss of proteostasis, increased cellular senescence, and stem cell exhaustion may contribute to the development of $\mathrm{AD}$ pathology and neurodegeneration (45). Identification of biomarkers of aging and elucidation of how the molecular pathways of aging and $\mathrm{AD}$ intersect could advance the identification of novel therapeutic targets and next-generation therapies, such as the use of mesenchymal stem cells (46). The links between aging and AD are being explored as one element of the INSPIRE Research Initiative (Barreto JFA in press).

\section{Conclusions/moving forward}

While the AD drug development pipeline continues to be dominated by $A \beta$-targeting therapies, there is increasing recognition that addressing the complexity of $\mathrm{AD}$ may require multiple agents and may need to start in early disease stage before pathology becomes irreversible. A "deep biology" view, such as that proposed by advocates of p75NTR modulation, posits that key 'hub' targets may enable modulation of multiple mechanisms (e.g. resilience to both $A \beta$ and tau) and that key components of pathology could be reversible (e.g. spines, synaptic function). A single treatment could thus promote synaptic function and slow progression and prevent upstream tau aggregation and oligomer formation.

Given the importance of tau in the development of AD, and reflecting the recently proposed Research Framework (47), CTAD Task Force members advocated assessment of both $\mathrm{A} \beta$ and tau levels in all clinical trials. The $\mathrm{A}-\mathrm{T}+\mathrm{N}+$ AD phenotype is common and should be targeted for anti-tau trials. A suggestion was made to name this phenotype Dementia Associated and Neurofibrillary tangle Neuroimaging Abnormality (DANNA). Tau imaging may provide a biological outcome, at least in Phase 2 studies, although the Task Force recognized that amyloid and/or tau PET imaging adds substantial subject and trial burden and cost. Other suggestions that could accelerate the development of anti-tau therapies include using basket designs that include participants with other tauopathies such as frontotemporal degeneration (FTD), progressive supranuclear palsy (PSP), and corticobasal degeneration (CBD). While such trials would include participants with heterogeneous presentations, an outcome assessment such as Goal Attainment Scaling (GAS) could enable capture of clinically meaningful outcomes from diverse participants. This tool enables patients, caregivers, and clinicians, to set goals for treatment using a standardized guided interview, followed by an assessment of whether those goals have been attained $(48,49)$. 
The Task Force suggested that combination therapy may be required to tackle such a complex disease as $\mathrm{AD}$ (50). They also advocated employing other innovative clinical trial methodologies to accelerate development of alternative approaches.

The Task Force proposed a general strategy to accelerate the development of alternative treatment approaches, which would include:

- Increased partnerships in the pre-competitive space with increased sharing of granular level data, shared biomarkers, statistical approaches, information on site performance

- Innovative trial design

- More collaborative approaches to recruitment and retention of participants for clinical trials with a focus on participation of representative populations.

Acknowledgements: The authors thank Lisa J. Bain for assistance in the preparation of this manuscript.

Conflicts of interest: The Task Force was partially funded by registration fees from industrial participants. These corporations placed no restrictions on this work. Dr. Gauthier is a member of scientific advisory boards for Biogen, Boehringer-Ingelheim, and TauRx; and a member of the DSMB for ADCS, ATRI, and Banner Health; Dr. Aisen reports grants from Janssen, grants from NIA, grants from FNIH, grants from Alzheimer's Association, grants from Eisai, personal fees from Merck, personal fees from Biogen, personal fees from Roche, personal fees from Lundbeck, personal fees from Proclara, personal fees from Immunobrain Checkpoint, outside the submitted work; Dr. Cummings is a consultant for Acadia, Actinogen, AgeneBio, Alkahest, Alzheon, Annovis, Avanir, Axsome, Biogen, Cassava, Cerecin, Cerevel, Cognoptix, Cortexyme, EIP Pharma, Eisai, Foresight, Gemvax, Green Valley, Grifols, Karuna, Nutricia, Orion, Otsuka, Probiodrug, ReMYND, Resverlogix, Roche, Samumed, Samus Therapeutics, Third Rock, Signant Health, Sunovion, Suven, United Neuroscience pharmaceutical and assessment companies, and the Alzheimer Drug Discovery Foundation; and owns stock in ADAMAS, BioAsis, MedAvante, QR Pharma, and United Neuroscience. Dr. Detke reports personal fees, non-financial support and other from Cortexyme, during the conduct of the study; personal fees and other from Embera, personal fees and other from Evecxia, personal fees from NIH, outside the submitted work; Dr Kramer is an employee of Eisai Company, Ltd; Dr Longo has equity in and consults for PharmatrophiX, a company focused on the development of small molecule modulators for neurotrophin receptors. He is also a co-inventor on related patent applications. Dr. Raman reports grants from NIH, grants from Eli Lilly, grants from Eisai, outside the submitted work; Dr Sabbagh reports personal fees from Allergan, personal fees from Biogen, personnal fees from Grifols, personal fees from vTV Therapeutics, personal fees from Sanofi, personal fees from Neurotrope, personal fees from Cortexyme, other from Neurotrope, other from uMethod, other from Brain Health Inc, other from Versanum Inc, other from Optimal Cognitive Health Company, outside the submitted work; Dr. Schneider reports grants and personal fees from Eli Lilly, personal fees from Avraham, Ltd, personal fees from Boehringer Ingelheim, grants and personal fees from Merck, personal fees from Neurim, Ltd, personal fees from Neuronix, Ltd, personal fees from Cognition, personal fees from Eisai, personal fees from Takeda, personal fees from vTv, grants and personal fees from Roche/Genentech, grants from Biogen, grants from Novartis, personal fees from Abbott, grants from Biohaven, grants from Washington Univ/ NIA DIAN-TU, personal fees from Samus, outside the submitted work; Dr. Tanzi is a consultant and shareholder in AZTherapies, Amylyx, Promis, Neurogenetic Pharmaceuticals, Cerevance, and DRADS Capital; Dr. Tariot reports personal fees from Acadia, personal fees from AC Immune, personal fees from Axsome, personal fees from BioXcel, personal fees from Boehringer-Ingelheim, personal fees from Brain Test Inc., personal fees from Eisai, personal fees from eNOVA, personal fees from Gerontological Society of America, personal fees from Otuska \& Astex, personal fees from Syneos, grants and personal fees from Abbvie, grants and personal fees from Avanir, grants and personal fees from Biogen, grants and personal fees from Cortexyme, grants and personal fees from Genentech, grants and personal fees from Lilly, grants and personal fees from Merck \& Co, grants and personal fees from Roche, grants from Novartis, grants from Arizona Department of Health Services, grants from National Institute on Aging, other from Adamas, outside the submitted work; In addition, Dr. Tariot has a patent U.S. Patent \# 11/632,747, "Biomarkers of Neurodegenerative disease." issued; Dr. Weiner is the PI of The Alzheimer's Disease Neuroimaging Initiative (ADNI) and the Brain Health Registry. I am a Professor at University of California San Francisco; Dr. Touchon has received personnal fees from Regenlife and is JPAD associated Editor and part of the CTAD organizing committee; Dr. Vellas reports grants from Lilly, Merck, Roche,
Lundbeck, Biogen, grants from Alzheimer's Association, European Commission, personal fees from Lilly, Merck, Roche, Biogen, outside the submitted work

Open Access: This article is distributed under the terms of the Creative Commons Attribution 4.0 International License (http://creativecommons.org/ licenses/by/4.0/), which permits use, duplication, adaptation, distribution and reproduction in any medium or format, as long as you give appropriate credit to the original author(s) and the source, provide a link to the Creative Commons license and indicate if changes were made.

\section{References}

1. Aisen, PS, Cummings, J, Doody, R, Kramer, L, Salloway, S, et al. The Future of Anti-Amyloid Trials. J Prev Alz Dis 2020;3(7):146-151.

2. Kunkle BW, Grenier-Boley B, Sims R, Bis JC, Damotte V, Naj AC, et al. Genetic meta-analysis of diagnosed Alzheimer's disease identifies new risk loci and implicates A $\beta$, tau, immunity and lipid processing. Nat Genet. 2019;51(3):414 30 .

3. Suh J, Choi SH, Romano DM, Gannon MA, Lesinski AN, Kim DY, et al ADAM10 missense mutations potentiate $\beta$-amyloid accumulation by impairing prodomain chaperone function. Neuron. 2013 Oct 16;80(2):385-401.

4. Jansen IE, Savage JE, Watanabe K, Bryois J, Williams DM, Steinberg S, et al. Genome-wide meta-analysis identifies new loci and functional pathways influencing Alzheimer's disease risk. Nat Genet. 2019;51(3):404-13.

5. Long JM, Holtzman DM. Alzheimer Disease: An Update on Pathobiology and Treatment Strategies. Cell. 2019 Oct 3;179(2):312-39.

6. Schneider JA, Arvanitakis Z, Bang W, Bennett DA. Mixed brain pathologies account for most dementia cases in community-dwelling older persons. Neurology. 2007 Dec 11;69(24):2197-204.

7. Cummings J, Lee G, Ritter A, Sabbagh M, Zhong K. Alzheimer's disease drug development pipeline: 2019. Alzheimers Dement (N Y). 2019;5:272-93.

8. Congdon EE, Sigurdsson EM. Tau-targeting therapies for Alzheimer disease. Nat Rev Neurol. 2018 Jul;14(7):399-415.

9. Digma LA, Madsen JR, Reas ET, Dale AM, Brewer JB, Banks SJ, et al. Tau and atrophy: domain-specific relationships with cognition. Alzheimer's Research \& Therapy. 2019 Jul 27;11(1):65.

10. Schwarz AJ, Yu P, Miller BB, Shcherbinin S, Dickson J, Navitsky M, et al. Regional profiles of the candidate tau PET ligand 18 F-AV-1451 recapitulate key features of Braak histopathological stages. Brain. 2016 May 1;139(5):1539 50 .

11. Gallardo G, Wong CH, Ricardez SM, Mann CN, Lin KH, Leyns CEG, et al. Targeting tauopathy with engineered tau-degrading intrabodies. Mol Neurodegener. 2019 22;14(1):38.

12. Hanseeuw BJ, Betensky RA, Jacobs HIL, Schultz AP, Sepulcre J, Becker JA, et al. Association of Amyloid and Tau With Cognition in Preclinical Alzheimer Disease: A Longitudinal Study. JAMA Neurol. 2019 Jun 3;

13. Zetterberg H, Burnham SC. Blood-based molecular biomarkers for Alzheimer's disease. Molecular Brain. 2019 Mar 28;12(1):26.

14. Eisenberg D, Jucker M. The amyloid state of proteins in human diseases. Cell. 2012 Mar 16;148(6):1188-203.

15. Longo FM, Massa SM. Small-molecule modulation of neurotrophin receptors: a strategy for the treatment of neurological disease. Nat Rev Drug Discov. 2013 Jul;12(7):507-25.

16. Knowles JK, Rajadas J, Nguyen T-VV, Yang T, LeMieux MC, Vander Griend $\mathrm{L}$, et al. The p75 neurotrophin receptor promotes amyloid-beta(1-42)-induced neuritic dystrophy in vitro and in vivo. J Neurosci. 2009 Aug 26;29(34):1062737.

17. Counts SE, He B, Prout JG, Michalski B, Farotti L, Fahnestock M, et al. Cerebrospinal Fluid proNGF: A Putative Biomarker for Early Alzheimer's Disease. Curr Alzheimer Res. 2016;13(7):800-8.

18. Yang T, Knowles JK, Lu Q, Zhang H, Arancio O, Moore LA, et al. Small molecule, non-peptide p75 ligands inhibit Abeta-induced neurodegeneration and synaptic impairment. PLoS ONE. 2008;3(11):e3604.

19. Xie Y, Meeker RB, Massa SM, Longo FM. Modulation of the p75 neurotrophin receptor suppresses age-related basal forebrain cholinergic neuron degeneration. Sci Rep. 2019 Mar 27;9(1):5273.

20. Nguyen T-VV, Shen L, Vander Griend L, Quach LN, Belichenko NP, Saw N et al. Small molecule p75NTR ligands reduce pathological phosphorylation and misfolding of tau, inflammatory changes, cholinergic degeneration, and cognitive deficits in $\mathrm{A} \beta \mathrm{PP}(\mathrm{L} / \mathrm{S})$ transgenic mice. J Alzheimers Dis. 2014;42(2):459-83.

21. Boros BD, Greathouse KM, Gentry EG, Curtis KA, Birchall EL, Gearing M, et al. Dendritic spines provide cognitive resilience against Alzheimer's disease. Ann Neurol. 2017 Oct;82(4):602-14.

22. Simmons DA, Belichenko NP, Ford EC, Semaan S, Monbureau M, Aiyaswamy $\mathrm{S}$, et al. A small molecule p75NTR ligand normalizes signalling and reduces Huntington's disease phenotypes in R6/2 and BACHD mice. Hum Mol Genet. 2016 15;25(22):4920-38.

23. Elshaer SL, Alwhaibi A, Mohamed R, Lemtalsi T, Coucha M, Longo FM, et al. Modulation of the p75 neurotrophin receptor using LM11A-31 prevents diabetes-induced retinal vascular permeability in mice via inhibition of 
inflammation and the RhoA kinase pathway. Diabetologia. 2019;62(8):1488500.

24. Shi J, Longo FM, Massa SM. A small molecule p75(NTR) ligand protects neurogenesis after traumatic brain injury. Stem Cells. 2013 Nov;31(11):256174.

25. Soscia SJ, Kirby JE, Washicosky KJ, Tucker SM, Ingelsson M, Hyman B, et al. The Alzheimer's disease-associated amyloid beta-protein is an antimicrobial peptide. PLoS ONE. 2010 Mar 3;5(3):e9505.

26. Moir RD, Lathe R, Tanzi RE. The antimicrobial protection hypothesis of Alzheimer's disease. Alzheimers Dement. 2018;14(12):1602-14.

27. Seo D-O, Holtzman DM. Gut microbiota: from the forgotten organ to a potential key player in the pathology of Alzheimer disease. J Gerontol A Biol Sci Med Sci. 2019 Nov 18;

28. Dominy SS, Lynch C, Ermini F, Benedyk M, Marczyk A, Konradi A, et al. Porphyromonas gingivalis in Alzheimer's disease brains: Evidence for disease causation and treatment with small-molecule inhibitors. Sci Adv. 2019;5(1):eaau3333.

29. Tzeng N-S, Chung C-H, Lin F-H, Chiang C-P, Yeh C-B, Huang S-Y, et al. Antiherpetic Medications and Reduced Risk of Dementia in Patients with Herpes Simplex Virus Infections-a Nationwide, Population-Based Cohort Study in Taiwan. Neurotherapeutics. 2018;15(2):417-29.

30. Howard R, Zubko O, Bradley R, Harper E, Pank L, O'Brien J, et al. Minocycline at 2 Different Dosages vs Placebo for Patients With Mild Alzheimer Disease: A Randomized Clinical Trial. JAMA Neurol. 2019 Nov 18;

31. Zhang C, Griciuc A, Hudry E, Wan Y, Quinti L, Ward J, et al. Cromolyn Reduces Levels of the Alzheimer's Disease-Associated Amyloid $\beta$-Protein by Promoting Microglial Phagocytosis. Sci Rep. 2018 18;8(1):1144.

32. Livingston G, Sommerlad A, Orgeta V, Costafreda SG, Huntley J, Ames D, et al. Dementia prevention, intervention, and care. The Lancet. 2017 Dec 16;390(10113):2673-734.

33. Williamson JD, Pajewski NM, Auchus AP, Bryan RN, Chelune G, Cheung AK, et al. Effect of Intensive vs Standard Blood Pressure Control on Probable Dementia: A Randomized Clinical Trial. JAMA. 2019 Feb 12;321(6):553-61.

34. Rouch L, Cestac P, Hanon O, Ruidavets J-B, Ehlinger V, Gentil C, et al. Blood pressure and cognitive performances in middle-aged adults: the Aging, Health and Work longitudinal study. J Hypertens. 2019 Jun;37(6):1244-53.

35. Ngandu T, Lehtisalo J, Solomon A, Levälahti E, Ahtiluoto S, Antikainen R, et al. A 2 year multidomain intervention of diet, exercise, cognitive training, and vascular risk monitoring versus control to prevent cognitive decline in at-risk elderly people (FINGER): a randomised controlled trial. Lancet. 2015 Jun 6;385(9984):2255-63.

36. Andrieu S, Guyonnet S, Coley N, Cantet C, Bonnefoy M, Bordes S, et al. Effect of long-term omega 3 polyunsaturated fatty acid supplementation with or without multidomain intervention on cognitive function in elderly adults with memory complaints (MAPT): a randomised, placebo-controlled trial. Lancet Neurol. 2017 May;16(5):377-89.

37. Vellas B, Carrie I, Gillette-Guyonnet S, Touchon J, Dantoine T, Dartigues JF, et al. MAPT Study: A multidomain approach for preventing Alzheimer's disease: Design and baseline date. J Prev Alzheimers Dis. 2014 Jun;1(1):13-22. 38. Hooper C, De Souto Barreto P, Coley N, Cantet C, Cesari M, Andrieu S, et al. Cognitive Changes with Omega-3 Polyunsaturated Fatty Acids in NonDemented Older Adults with Low Omega-3 Index. J Nutr Health Aging. 2017;21(9):988-93.

39. Delrieu J, Payoux P, Carrié I, Cantet C, Weiner M, Vellas B, et al. Multidomain intervention and/or omega-3 in nondemented elderly subjects according to amyloid status. Alzheimers Dement. 2019 Sep 23;

40. Chhetri JK, de Souto Barreto P, Cantet C, Pothier K, Cesari M, Andrieu S, et al. Effects of a 3-Year Multi-Domain Intervention with or without Omega-3 Supplementation on Cognitive Functions in Older Subjects with Increased CAIDE Dementia Scores. J Alzheimers Dis. 2018;64(1):71-8.

41. Rosenberg A, Mangialasche F, Ngandu T, Solomon A, Kivipelto M. Multidomain interventions to prevent cognitive impairment, alzheimer's disease, and dementia: from finger to world-wide fingers. Journal of Prevention of Alzheimer's Disease [Internet]. 2019 Mar 1 [cited 2020 Jan 30]; Available from: http:/ / www.jpreventionalzheimer.com/all-issues.html

42. Blivet G, Meunier J, Roman FJ, Touchon J. Neuroprotective effect of a new photobiomodulation technique against A $325-35$ peptide-induced toxicity in mice: Novel hypothesis for therapeutic approach of Alzheimer's disease suggested. Alzheimers Dement (N Y). 2018;4:54-63.

43. Lin Y, Jiang W-J, Shan P-Y, Lu M, Wang T, Li R-H, et al. The role of repetitive transcranial magnetic stimulation (rTMS) in the treatment of cognitive impairment in patients with Alzheimer's disease: A systematic review and meta-analysis. J Neurol Sci. 2019 Mar 15;398:184-91.

44. Sierra F. Geroscience and the role of aging in the etiology and management of alzheimer's disease. Journal of Prevention of Alzheimer's Disease [Internet]. 2019 Mar 1 [cited 2020 Jan 30]; Available from: http:/ /www. jpreventionalzheimer.com/all-issues.html

45. Guerville F, De Souto Barreto P, Ader I, Andrieu S, Casteilla L, Dray C, et al. Revisiting the Hallmarks of Aging to Identify Markers of Biological Age. J Prev Alzheimers Dis. 2020;7(1):56-64

46. Oliva AA, McClain-Moss L, Pena A, Drouillard A, Hare JM. Allogeneic mesenchymal stem cell therapy: A regenerative medicine approach to geroscience. Aging Med (Milton). 2019 Sep;2(3):142-6.

47. Jack CR, Bennett DA, Blennow K, Carrillo MC, Dunn B, Haeberlein SB, et al. NIA-AA Research Framework: Toward a biological definition of Alzheimer's disease. Alzheimers Dement. 2018;14(4):535-62.

48. Rockwood K, Graham JE, Fay S, ACADIE Investigators. Goal setting and attainment in Alzheimer's disease patients treated with donepezil. J Neurol Neurosurg Psychiatry. 2002 Nov;73(5):500-7.

49. Rockwood K, Fay S, Song X, MacKnight C, Gorman M. Attainment of treatment goals by people with Alzheimer's disease receiving galantamine: a randomized controlled trial. CMAJ. 2006 Apr 11;174(8):1099-105.

50. Gauthier S, Alam J, Fillit H, Iwatsubo T, Liu-Seifert H, Sabbagh M, et al. Combination Therapy for Alzheimer's Disease: Perspectives of the EU/US CTAD Task Force. J Prev Alzheimers Dis. 2019;6(3):164-8. 